\title{
Improving Efficiency in Organizations by Monitoring Stress and Promoting Awareness and Wellbeing at the Workplace
}

\author{
Tiago Nascimento and Gabriel Pestana \\ Universidade Europeia, Lisboa, Portugal \\ tiago.filipedonascimento@gmail.com \\ gabriel.pestana@universidadeeuropeia.com \\ DOI: 10.34190/KM.19.165
}

\begin{abstract}
Stress at the workplace has been associated with an increase in absenteeism and presenteeism in organisations, with a high impact regarding productivity and co-worker's wellbeing at the workplace. The paper addresses such concerns by considering an approach that can act preventively by implementing a framework for early detection of stressing symptoms. The aim is to provide the organisation with a situational-awareness tool to keep the decision-maker well informed about any suspicious situation requiring the user attention, providing insights based on the co-worker wellness and specific needs, prompting in this way a healthy policy environment at the workplace. The research challenge addresses observing coworkers based on parameters associated with health indicators and understands how their physical and mental behaviour at the workplace can be affected by stress levels, including studying the impact derived from the conditions provided at the workplace environment. The paper presents an ongoing research work for early recognition of the identified risk factors, and to engage the workers in becoming proactive in their workplace. The primary goal of this model is to monitor the risk factors with impact on managing stress at the workplace. From a management viewpoint, the proposed model addresses the general theory of systems, as each variable in a system interacts with other variables so completely that cause and effect cannot be separated, as the workplace and the collaborators are linked together in order to maintain an equilibrium as is the best efficiency and efficacy at organizations. We expect to develop an integrated, systematic and dynamic model to support decision-makers (e.g., health supervisor) with the embedded knowledge required to choose the best possible intervention for the maximum benefit of the client (i.e., co-worker). The Boyd Cycle complements the decision-making process in order for health professionals to make more informed decisions in useful time. The Boyd Cycle assumes the existence of constant feedback and reorientation based on existing information and intuition, promoting the management of the workers and environment information, leading to adjustments in the workplace in real-time. The research will adopt the Design Science Research Methodology (DSRM) approach, where each informational artefact is generated to address the challenges identified for the target group. The study object for the proposed model is the health sciences with a focus on the nursing discipline and will be validated using interviews, panels of experts and later with the use of simulations.
\end{abstract}

Keywords: stress and wellbeing determinants, preventive actions, absenteeism, situational-awareness, Boyd cycle

\section{Introduction}

The study of stress and wellbeing at the workplace has been an object of great interest within the scientific community (Taris, 2016; Bae et al., 2019), namely to understand its impact on the productivity of the company. The conditions in the workplace and the tasks that the employee has to perform are pointed out as the main determinants for absenteeism and presenteeism with impact to both from a financial perspective and in the company overall productivity. This paper presents undergoing research focused on analysing the determinants of stress and wellbeing at the workplace. The research challenge addresses the study of metabolic syndromes associated with unhealthy lifestyles and co-workers behaviour at the workplace, including proactively triggering awareness events to stakeholders (i.e., decision-makers) whenever a suspicious situation, requiring their intervention, is detected by the proposed surveillance model. The goal is to, act preventively in motivating employees to adopt work habits and healthier lifestyles at the workplace, in particular for jobs subject to recurrent stressing situations. The expected outcome is to reduce absenteeism and presentism in the company and, consequently, increase productivity and efficiency in the workplace with better-perceived working conditions.

According to EUROSTAT, in 2017 there were 227,630,000 citizens (79.7\% of which are full-time workers). In Portugal, the number of full-time workers is $4,756,000$, corresponding to $88.7 \%$. Considering that on average, a full-time worker will stay at the workplace for at least 8 hours, it is most important to attend employees comfort and wellness at work. Depending on the job function or responsibilities, the conditions provided at the workplace might influence employee's state of health, their productivity, including the emergence of complications related to chronic diseases that may exist. This is particularly relevant for two categories of professionals: 
- White-collar workers (WCW), individuals frequently exposed to job-related stressful factors, such as labour and occupational stressors (e.g., cognitive and mental tiredness, intense intellectual activity, decisionmaking tasks, teamwork coordination). This might harm their physical and emotional wellbeing.

- Blue-collar workers (BCW), indicating less qualified workers and jobs pointing to repetitive physical/operational tasks/decisions and in this case, aiming to identify behavioural patterns caused by repetitive tasks (e.g., tasks requiring some level of concentration for long shifts, or disorders caused by high physical loads).

An absenteeism at the workplace is usually associated with missing work because of justified situations (e.g., sickness or any other consented absence) or because of unjustified situations, namely: extended (lunch) breaks, nonattendance (during long periods or systematically for short periods) during work hours or any other absence not formally approved by the employer (Munro, 2007). In Europe, the average absence rate relies upon between $3 \%$ and $6 \%$ of the working hours, corresponding to a global cost of $2.5 \%$ of GDP (EUROSTAT, 2017).

From a healthcare perspective, workplace conditions may include additional risk factors that might affect the workers' wellbeing or satisfaction in the workplace. This is particularly relevant for chronic or acute diseases; in this domain, the culture and social policies of the organisation can make the difference in dealing with this type of concerns (Brooks et al., 2010; Brown et al., 2011). The promotion of wellness in the workplace might change the determinants of health, promoting a better quality of life, empowering workers to be more proficient in doing their job (Eurofound, 2010). Among the determinants of health, healthy lifestyles are one of the determinants that provide better health gains.

According to George (2004), "the citizen plays an active role in the adoption of healthy lifestyles, in close collaboration with the health professional." Such active and responsive citizens should be recognised and promoted as good examples. This type of behaviours represents a benefit to the company performance health and sustainability; such responsive citizens should treat as the driving force behind the change that is required for the sustainability of the national healthcare system. The current economist view of clients (i.e., patients) of the healthcare system needs to change. It has to take into account those workers who act proactively, investing in healthy lifestyles from those who irresponsibly persist with unhealthy habits or, from the employer perspective, alert and inform companies without any perceived concern in providing better conditions in the workplace. Such approach streamlines the implementation of ground-breaking innovations regarding selfawareness, contributing simultaneously to the adoption of corporate situational-awareness programs that can act preventively in detecting symptoms or any other risk factor of stress in the workplace.

The concept of situational-awareness was defined by Endsley (1995) as the perception of the elements in the environment within a volume of time and space, the comprehension of their meaning and the projection of their status shortly. The concept is segmented into the following three components: perception, comprehension, and projection. The perception is the lowest level of situational awareness, without data interpretation, just the receipt of the information. The comprehension follows the perception of the elements to produce an understanding of the information; the degree of comprehension depends on the person's expertise. Finally, the projection is the highest level of situational awareness and is the capacity to project the future, considering the elements at disposal. This anticipation will provide time to solve the problems before they get installed. So, this highest level of situational awareness is expected to be developed in the co-workers through time passed in the organisation.

The paper presents the following structure. Section 2 presents a detailed discussion about stress and wellbeing; Section 3 explains the elements of the proposed model and how it is structured. Finally, in section 4 , presents the conclusions and the challenges for future work.

\section{Stress and well being}

The World Health Organization (WHO, 1948) defines health as a state of complete physical, mental and social wellbeing and not merely the absence of disease or infirmity. In this way, physical and mental wellbeing must be constant throughout life, encompassing not only the perspective at home but also a continuity in the work context. In 2014, WHO inquiry or mental health as a condition for the existence of a global wellbeing, especially in the employment context, defining it as a welfare state in which every individual realizes his potential, can cope with the usual life tensions, can work productively and fruitfully and is able to contribute to their community (WHO, 2014). Considering the high prevalence of stress in the workplace is necessary to define 
stress, which has been established in different ways over the years - designed as ambient pressure (Suarez, 2011) and as tension within the person (Santos and Castro, 1998). The commonly acceptable and today is the interaction between the state and the individual. It is the psychological and physical state that results when the resources of the individual are not enough to deal with the demands and pressures of the situation (Abreu et al., 2002).

Stress can affect the achievement of goals, both for the employee and the company (Michie, 2002). Although the approach to stress is also necessary to list the positive perspective of stress, that is, how that stress can have a positive impact on the employment context, so this positive stress should be one of the goals to achieve within the organisation, promoting this feeling of wellbeing in the co-workers. The positive stress, designated as eustress (Quick et al., 2003), is associated with excellent performance and health outcomes. Distress arises as a stress to the negative impact, i.e., pathological stress, which affects the overall wellbeing (Mckenzie and Harris, 2013). This stress can be developed by changes in the employment context or the workplace, i.e., the variables related to working conditions and work organisation. Both the quantitative workload (the amount of work to be done) and the qualitative workload (work difficulty) is associated with stress. The workload must be measured considering the relationship with the workspace, i.e., the speed at which the work should be performed and the nature and control of the stimulation requirements.

The content of the work (or task design) includes various aspects to be monitored, such as low work value, low skill use, lack of variety of tasks and repeatability at work, uncertainty, lack of opportunity to learn and insufficient resources (Nord et al., 1990). In addition to the content of the work, it is also essential for the mental wellbeing, the work contexts, which include psychosocial concerns and risks in the organization of work and labor relations, such as culture and organizational function, role in the company, development of career, decision-latitude and control, homework interface and interpersonal relationships at work. Aspects of culture and corporate function are particularly significant: organisation as a task performance environment, as a problem-solving environment, and as an accessible development environment. An organisation with such characteristics is plausible to achieve increased levels of stress. In this field, organisational functions where role ambiguity and role conflict persist, role overload, role failure and accountability for other people are often referred to as distress enablers. Paper failure (when the individual skills and training are not fully used) is frequently associated with low satisfaction levels are at work and low organisational commitment (ILO, 2016). In this way, the creation of a model for monitoring all these variables will allow, in a preventive way, to promote the wellbeing of employees, intervening early in situations that may trigger processes of presenteeism and, later, absenteeism. Therefore, the proposed model is in line with current research in addressing the efficiency of organisations. This model may also lead to a change in organisational policies and, from a macro perspective, a change in health and environmental policies (Deloitte Centre for health solutions, 2017). By promoting workplaces where employees are healthy and happy, these tend to be more productive, valuing a correct balance between personal and professional life and leading to the maintenance of employees in companies, reducing turnover and associated direct and indirect costs $(\mathrm{CBI}, 2018)$. If these problems are unrecognised or neglected, this may lead to a range of potentially undesirable behaviours and a decrease in productivity.

Therefore, we can say that an organisation works as a living system, so we based our model in Systems Theory, specifically in General Systems Theory, developed by Ludwig von Bertalanffy (1968). This theory conceptualises that a system cannot be reduced separate parts working separately but, if we want to understand the whole, we need to understand the relations and interdependencies between those parts. In a management perspective, "application of this theory rests on the assumption that most individuals strive to do good work, but that they are acted upon by diverse influences, and that functional and efficient systems not only account for but also embrace these influences" (Anderson, 2016). Considering the organisation as a system, we can see the "systems thinking is an approach or a methodology for addressing problems. It follows two basic premises, which include looking at reality in terms of wholes, and acknowledging that the environment is an essential part of the system, as it interacts with the system" (Kramer and De Smit, 2012). Cordon (2013) tells us that the challenge is to identify the different elements and units, large or small that affect way care is provided to patients (workers in this setting). Thinking this way will promote a better development in the organisations, converting them in learning organisations. Also, this model can help the organization to make decisions in every level, not only in the operational level, i.e., the short-term decision making related to the execution of the health care delivery process but also at tactical level, strategic planning addresses structural decision making, tactical planning addresses the organization of the operations/execution of the health care delivery process (Hall, 2012). 


\section{Preventive monitoring and diagnosis model}

The Preventive Monitoring and Diagnosis (MDP) model presented in this article, is based on the use of the Boyd Cycle, as known as OODA Loop (Thompson, 1995; Osinga, 2007), seen as a decision-making model, but it can be more accurately described as a model of learning and individual and organizational adaptation. The OODA Loop facilitates decision making because it is filtered through culture, genetics, previous experience, new information, and the ability to analyse and synthesise (Enck, 2012). It aims at integral and systemic monitoring of the collaborator in the work context, integrating both analysis dimensions typical of work-content and work-context, as well as monitoring of the organisation itself, allowing the creation of a space that promotes the physical and mental wellbeing. As a management tool, the OODA Loop can be compared with the Demming Cycle, as known as PDCA (Plan-Do-Check-Act), used first for quality control and then to measure the performance of the organisations. The problem is that PDCA lacks the learning process and the constant adaptation (Grant and Kooter, 2005). Within the scope of the project, two categories of professionals will be considered for the targetgroup: white-collar workers (WCW) and blue-collar workers (BCW), the BCW can be defined as those who perform primarily physical work and whose career paths are relatively restricted and WCW professional and semi-professional employees (Hu, Kaplan and Dalal, 2010).

Presently global healthcare is being hampered by information chaos, which in turn leads to inferior decisionmaking, ineffective and inefficient operations, exponentially increasing costs and even loss of life. To alleviate such predicament, the merits of adopting and adapting the doctrine of network-centric operations to healthcare has been recommended (Lubitz and Wickramasinghe, 2006).

The proposed model has as a central element a client manager who, continuously, receives notifications whenever its intervention is requested. The client manager has awareness mechanisms expressed through informational articles that help identify when it has intervened $r$, for example, to respond to deviations that indicate the risk associated with an increased level of stress or low comfort $d$ or collaborator in the labour context. These artefacts correspond to graphical indicators in an interactive dashboard interface. The manager also commonly has diagnostic mechanisms to speed up the decision process and may consult Standard operating procedures (Standard Operating Procedures - SOP) (Gough et al., 2009) suggested by the model against the input parameters of the generated diagnosis. Boyd Cycle will allow the manager to make decisions in real-time, with as many data as possible.

The cycle presupposes the existence of constant feedback and reorientation, based on decision making, external information and intuition, promoting a continuum with a view to an equilibrium that allows the model to be integrated, systematic and dynamic in maximizing the benefit to the employee and the consequent impact in terms of productivity and wellbeing in the workplace.

The Boyd's OODA Loop provides an integrated, systematic and dynamic model for ensuring that the healthcare decision-maker is always provided with the appropriate and necessary knowledge elements that will help to ensure that healthcare decision making process outcomes are optimised for the maximal patient benefit (Wickramasinghe et al., 2009). This monitoring will be done with the support of Key Performance Indicators (KPI's), that can be defined as a set of measures focusing on those sides of organisational performance that are critical for the success of the organisations (Badawy et al., 2016). The same authors remember us that creating useful leading KPIs is essential to the success of any business organisation so that it is smart to changes quickly, and is prepared for the coming changes.

Table 1 presents the set of operational indicators required to cope with the situational-awareness mechanism required for continuum surveillance addressing preventive actions. The operational indicators express in numbers perceptions that would be otherwise difficult to identify and correlate (Arora and Kaur, 2015); therefore the indicators are grouped into three categories: Indicators of Physical Environment (work context), Indicators of Social Environment (work content) and Worker Health Context.

Monitoring of the physical environment is essential for reducing stress in the organisation. This dimension of analysis should have as reference improvement of comfort, aims to alert to situations that compromise, for example, ergonomic comfort, acoustic, thermal, and luminosity. Through the input data, the diagnosis recommended by the SOP should address economically viable solutions optimised for the highest possible comfort. We used the "Office Environment Model" (Bluyssen, Aries and Dommelen, 2011), which covers the 
various aspects of the workplace and its relation to stress. These authors define that the workplace should not cause or aggravate any illness of employees who occupy the building.

Table 1: Indicators to monitor in the model

\begin{tabular}{|c|c|c|}
\hline Categories & Perspective & Dimension of Analysis \\
\hline \multirow{8}{*}{$\begin{array}{c}\text { Work Context/Physical } \\
\text { Environment }\end{array}$} & \multirow{2}{*}{ Health and Comfort } & Health \\
\hline & & Comfort \\
\hline & \multirow{2}{*}{ Safety and Security } & Safety \\
\hline & & Security \\
\hline & \multirow{2}{*}{ Usability and Positive Stimulation } & Usability \\
\hline & & Positive Stimulation \\
\hline & \multirow{2}{*}{ Adaptability and Serviceability } & Adaptability \\
\hline & & Serviceability \\
\hline \multirow{3}{*}{ Work Content/Social Environment } & \multirow{2}{*}{ Workload and Demand } & Workload \\
\hline & & Demand \\
\hline & Occupational Consequences & Attendance \\
\hline \multirow{8}{*}{ Worker Health Context } & \multirow{6}{*}{ Physical Consequences } & Chronic Diseases \\
\hline & & Anthropometric Values \\
\hline & & Analytical Values \\
\hline & & Hospitalisation \\
\hline & & Changes in pain experience \\
\hline & & Physical Symptoms \\
\hline & \multirow{2}{*}{ Psychological Consequences } & Sleep Disturbances \\
\hline & & Psychological Disturbances \\
\hline
\end{tabular}

The monitoring of the social environment aims to measure, albeit subjectively, the workload of employees. This measurability allows associating the stress of the collaborator to his / her function within the organisation, also facilitating the distinction of other risk factors (physical or psychological) that underlie stress. For this, we used the "Task Load Index" model (Hart and Staveland, 1988), a multidimensional scale that, using six subscales, allows us to measure the workload of the collaborator.

These inputs will feed the dashboard into an operational and tactical perspective. The operational perspective refers to the inputs that will allow the promotion of situational awareness among employees, that is, data that will allow these employees to have information about the factors that induce stress. The tactical perspective allows the health professional, manager of the process, to evaluate and preventively mitigate the risk of distress in the employees, analysing whether it is an individual or systemic perspective.

The monitoring of these indicators, using sensors and feedback, allows and evaluates deviations based on evidence-based practice (i.e., Thresholds two indicators). The introduction of thresholds allows detecting deviations, activating alarm mechanisms for timely and effective intervention in risk prevention. This intervention will be parameterised using algorithms of diagnostics that will enable inferring what the best response to accomplish. It will be up to the model manager to decide on the best prescription of the intervention, previously proposed by the algorithm. This intervention will be supported on SOP's, which, in a systematised and standardised way, allow the response to be adjusted to the situation. If the model manager understands that an SOP is applied, he will prescribe it, initiating the intervention whose results, duly identified in time, will be shown in the dashboard. Lenert (2017) focuses that OODA loops emphasise recognition of mismatch between predictions and experiences and, also, automation of responses in specific settings, once a problem has been recognised. OODA and other models that formally integrate a predictive step enhance learning through observation. It also forces a projection on the learner and periodic (re) assessments to test that projection with reality. In Figure 1, it is explained the mode, and his information flows, supported in OODA Loop and with a focus on the separation of eustress and distress. This model begins with the Observ moment, with focus on all the KPI's monitored that will serve as an input to make the best decision. This data will then be 
integrated, analysed and therefore shown on the interactive dashboard, promoting the situational awareness, in both operational and tactical levels of decision. This will correspond to the Orient phase of the OODA Loop. Therefore, the model will, considering the information given, infer on the best SOP to apply to solve the situation.

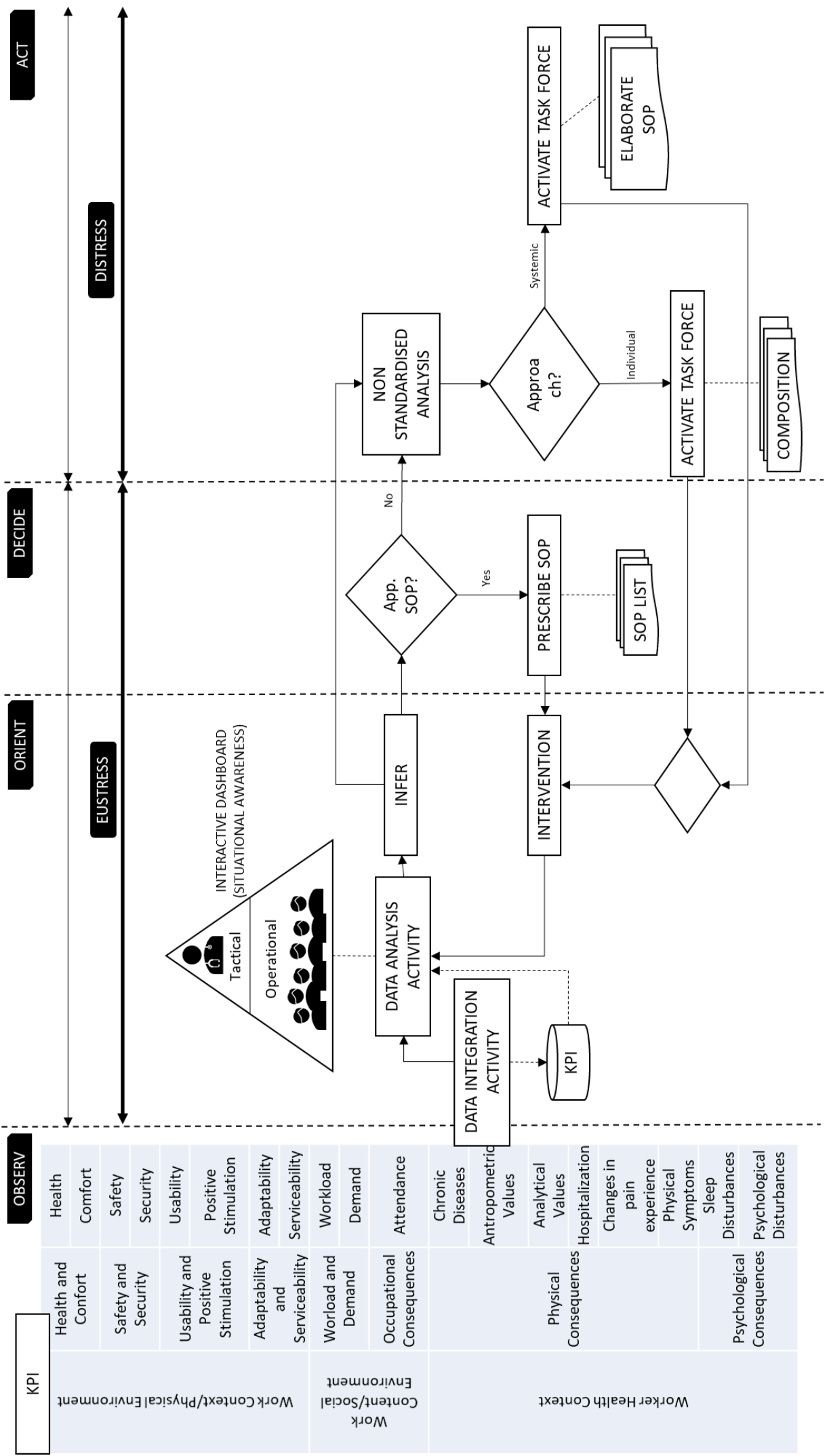

Figure 1: Preventive monitoring and diagnosis model 
If the manager decides to apply an SOP, he will prescribe it is recurring to an SOP list, proposed by the model and then began the intervention. These steps are considered at the eustress phase of a co-worker, solved without expecting any physical or phycological problems, deciding the best option to take. If any of this SOP's suggested applied, then the probability of the co-worker develops distress is higher, and the manager should take action, with a different approach, considering the need to implicate other persons in the process that will help to decide the best intervention and, simultaneously, develop a new SOP specifically for that situation.

If the manager considers that the proposed SOP does not fit into the alarm system, it should then prescribe a non-standardized intervention, which extends the perspective of an operational intervention, to a strategic response. This non-standardized analysis can thus be applied to an individual approach if it is focused on the employee and whose situation does not have a defined SOP, or from a systemic perspective. In this way, it is considered that risk mitigation from the perspective of work and organisation will bring added value in increasing productivity and efficiency, essential indicators for the management of organisations. For the success of the planned developments, the research adopts the Design Science Research Methodology approach (DSRM), in which each informational artefact is generated to address the challenges identified for the target group. The DSRM provides a theoretical framework, with a set of heuristics on good practices and procedures for designing innovative and ambitious systems. The result of this methodology will be the creation of an innovative device to solve specific problems within the health sector and be a contributor to organisations' decisions on an operational, and tactical level. As defended by Lapão, Mira and Gregório (2017), DSRM has demonstrated its ability to study the connection between research and professional practices by designing, implementing and evaluating artefacts that address a specific need. One of the biggest challenges is to identify leading indicators because it often hard requires months to collect needs, measure definitions and rules (Badawy et al., 2016).

\section{Conclusions and future work}

In conclusion, this model aims to mitigate the risk factors associated with the development of pathological stress, i.e. distress, by monitoring various indicators. Reducing distress in the workplace, coupled with promoting wellbeing, will lead to increased productivity.

If the target problem is not recognised or neglected, it can affect the productivity of the workers and the organisation in general. Standard operating procedures (SOPs) can help identify what actions need to be taken before it becomes critical, contributing to improving situational awareness within the organisation. Thus, the organisation will gain stability and an equilibrium between the environment and the workers, as seen by the general systems theory. The artefact derived from the application of DSRM is the testing of the proposed model, and the analysis of the results achieved from a filed survey. The health sciences focusing on the nursing discipline and will be validated through interviews, panels of experts and later with the use of simulations. It is expected that this model increases the efficiency of organisations, through a better outcome in work context, work content and workers' health. This model will also lead to an improvement in the political framework within the organisations and, expectedly, in all the health sector too.

\section{References}

Abreu, K. L. de et al. (2002) 'Estresse ocupacional e Síndrome de Burnout no exercício profissional da psicologia', Psicologia: Ciência e Profissão . scielo , pp. 22-29.

Anderson, B. R. (2016) 'Improving health care by embracing Systems Theory', Journal of Thoracic and Cardiovascular Surgery, 152(2), pp. 593-594. doi: 10.1016/j.jtcvs.2016.03.029.

Arora, A. and Kaur, S. (2015) 'Performance assessment model for management educators based on KRA/KPI', in International Conference on Technology and Business Management.

Badawy, M. et al. (2016) 'A survey on exploring key performance indicators', Future Computing and Informatics Journal. Elsevier Ltd, 1(1-2), pp. 47-52. doi: 10.1016/j.fcij.2016.04.001.

Bae, Y.-S. et al. (2019) 'Editorial: Stress and Immunity', Frontiers in Immunology, 10(February), pp. 1-2. doi: 10.3389/fimmu.2019.00245.

Von Bertalanffy, L. (1968) 'General System Theory', Georg. Braziller New York, 1, p. 289. Available at: http://books.google.es/books?id=N6k2mILtPYIC.

Bluyssen, P. M., Aries, M. and Dommelen, P. Van (2011) 'Comfort of workers in office buildings: The European HOPE project', Building and Environment. Elsevier Ltd, 46(1), pp. 280-288. doi: 10.1016/j.buildenv.2010.07.024.

Brooks, A. et al. (2010) 'Presenteeism: Critical Issues', JOEM, 52(11), pp. 1055-1067. doi: 10.1097/JOM.0b013e3181f475cc.

Brown, H. E. et al. (2011) 'Does Physical Activity Impact on Presenteeism and Other Indicators of Workplace Wellbeing?', Sports Med, 41(3), pp. 249-262. 
CBI (2018) Front of Mind: Prioritising Workplace Health and Wellbeing. Available at: http://www.cbi.org.uk/front-ofmind/recommendations.html (Accessed: 30 March 2019).

Cordon, C. P. (2013) 'System Theories: An Overview of Various System Theories and Its Application in Healthcare', American Journal of Systems Science, 2(1), pp. 13-22. doi: 10.5923/j.ajss.20130201.03.

Deloitte Centre for health solutions (2017) At a tipping point? Workplace mental health and wellbeing, Deloitte LLP.

Enck, R. E. (2012) 'The OODA Loop', pp. 1-2. doi: 10.1177/1084822312439314.

Endsley, M. R. (1995) 'Toward a Theory of Situation Awareness in Dynamic Systems', Human Error in Aviation, 37(December), pp. 217-249. doi: 10.4324/9781315092898-13.

Eurofound (2010) Absence from work A report based on the fifth European Working Conditions Survey. Available at: file://C:/Users/IMP2/Downloads/EF1302EN (3).pdf.

EUROSTAT (2017) Estatísticas do emprego - Statistics Explained. Available at: https://ec.europa.eu/eurostat/statisticsexplained/index.php?title=Employment_statistics/pt (Accessed: 25 March 2019).

George, F. (2004) 'Texto adaptado e resumido baseado num capítulo da publicação intitulada "Histórias de Saúde Pública " escrito pelo Autor e editado pelos Livros Horizonte (2004)', pp. 1-3.

Gough, J. et al. (2009) 'Standard Operating Procedures (SOPs): Why Companies Must Have Them, and Why They Need Them', Drug Information Journal. doi: 10.1177/009286150904300112.

Grant, T. and Kooter, B. (2005) 'Comparing OODA \& other models as Operational View C2 Architecture', in 10TH INTERNATIONAL COMMAND AND CONTROL RESEARCH AND TECHNOLOGY SYMPOSIUM.

Hall, R. (2012) Handbook of Healthcare System Scheduling. Edited by F. Hillier. New York: Springer Science \& Business Media. doi: 10.1007/978-1-4614-1734-7.

Hart, S. G. and Staveland, L. E. (1988) 'Development of NASA-TLX (Task Load Index): Results of empirical and theoretical research', in Advances in psychology. Elsevier, pp. 139-183.

$\mathrm{Hu}, \mathrm{X} .$, Kaplan, S. and Dalal, R. S. (2010) 'An examination of blue- versus white-collar workers' conceptualizations of job satisfaction facets', Journal of Vocational Behavior. Elsevier Inc., 76(2), pp. 317-325. doi: 10.1016/j.jvb.2009.10.014.

ILO (2016) Workplace Stress: A Collective Challenge, World Day for Safety and Health at Work. doi: 10.1017/CBO9781107415324.004.

Kramer, N. J. T. A. and De Smit, J. (2012) Systems thinking: Concepts and notions. Springer Science \& Business Media.

Lapão, L. V., Mira, M. and Gregório, J. (2017) 'Implementing an online pharmaceutical service using design science research', BMC Medical Informatics and Decision Making. BMC Medical Informatics and Decision Making, pp. 1-14. doi: 10.1186/s12911-017-0428-2.

Lenert, L. A. (2017) 'Toward Medical Documentation That Enhances Situational Awareness and Learning', AMIA Annu Symp Proc, (8), pp. 763-771.

Lubitz, D. Von and Wickramasinghe, N. (2006) 'Networkcentric Healthcare: outline of entry portal concept', International Journal of Electronic Business Management, 4(1), pp. 16-28.

Mckenzie, S. H. and Harris, M. F. (2013) 'Understanding the relationship between stress, distress and healthy lifestyle behaviour: a qualitative study of patients and general practitioners', BMC Family Practice.

Michie, S. (2002) 'Causes and Management of Stress At Work', Occupational and Environmental Medicine, 59(1), pp. 6772. doi: 10.1136/oem.59.1.67.

Munro, L. (2007) 'Absenteeism and presenteeism: possible causes and solutions .', THE SOUTH AFRICAN RADIOGRAPHER, 45(1), pp. 21-23.

Nord, W. R. et al. (1990) Studying meanings of work: The case of work values., Meanings of occupational work: A collection of essays. Lexington, MA, England: Lexington Books/D. C. Heath and Com (Issues in organization and management series.).

OMS (1948) Constitution. Available at: https://www.who.int/about/who-we-are/constitution (Accessed: 25 March 2019).

Osinga, F. P. B. (2007) Science, strategy and war: The strategic theory of John Boyd. Routledge.

Quick, James et al. (2003) 'Stress, health, and wellbeing at work', Organizational behavior: The state of the science (pp. 5389).

Santos, A. M. and Castro, J. J. (1998) 'Stress', Análise Psicológica, 4, pp. 675-690.

Suarez, C. (2011) 'Time Pressure and Attribution Effects in Stress Ratings', The Huron University College Journal of Learning and Motivation, 49(1).

Taris, T. W. (2016) 'Work \& Stress: Thirty years of impact', Work \& Stress. Taylor \& Francis, 8373. doi: 10.1080/02678373.2015.1137719.

Thompson, F. (1995) 'Business strategy and the Boyd cycle', Journal of Contingencies and Crisis Management. Wiley Online Library, 3(2), pp. 81-90.

WHO (2014) 'Mental health: a state of wellbeing', Mental health: a state of wellbeing. World Health Organization. Available at: https://www.who.int/features/factfiles/mental health/en/ (Accessed: 25 March 2019).

Wickramasinghe, N. et al. (2009) 'A systematic approach: optimization of healthcare operations with knowledge management.', Journal of healthcare information management : JHIM. United States, 23(3), pp. 44-50. 